\title{
A Research on Retinal Diseases Predictionin Image Processing
}

\author{
B.Sakthi Karthi Durai, J.Benadictraja, C.Sathya
}

\begin{abstract}
In this paper, a detail survey on retinal diseases identification using image processing is discussed. The retinal diseases are glaucoma, cataract, hypersensitive retinopathy, diabetic retinopathy, age related macular degeneration. The image processing provides greater innovation in medical applications. Image processing gives clear view to determine the infected area. Different algorithms and different procedure were followed here. The survey of detection in retinal diseases includes algorithms are elaborated and compared which are useful for researchers in image processing.This help the researcher get a detailed view of image processing in retinal diseases identification.
\end{abstract}

Keywords: Diabetic retinopathy, Age-related macular degeneration, hyper sensitive machine. retinopathy, Support vector

\section{INTRODUCTION}

Image processing is a strategy to change over a picture into advanced structure and play out a few tasks on it, so as to get an upgraded picture or to separate some valuable data from it. It is a sort of flag agreement in which input is picture, similar to video edge or photo and yield might be picture or attributes related with that picture. Commonly Image dealing system includes about pictures as two dimensional signs while applying adequately set flag preparing methods to them. It is among rapidly creating advancements today, with its applications in various fragments of a business. Picture Processing borders focus investigate about zone inside planning and programming building disciplines also. [1] Image processing is currently routinely utilized by a wide scope of people who approach advanced cameras and PCs. With a base speculation, one can promptly improve differentiate, identify edges, measure force, and apply an assortment of scientific activities to pictures.

Retinal illnesses differ generally, however the majority of them cause visual manifestations. Retinal ailments can influence any piece of your retina, a slight layer of tissue within back mass of your eye. The retina contains a huge number of light-touchy cells (bars and cones) and other nerve cells that get and compose visual data. Your retina sends this data to your mind through your optic nerve, empowering you to see.[2]

\section{IMAGE PROCESSING IN MEDICAL APPLICATION}

This exploration fuses auxiliary and practical MRI in nervous system science, examination of bone shape and structure, advancement of useful imaging devices in oncology, and PET picture handling programming improvement.

Malignant growth Imaging(cancer) - Different devices, for example, PET, MRI, and Computer supported Detection analyze and know about the tumor.

Cerebrum Imaging(brain) - Focuses on the typical and unusual improvement of mind, mind maturing and normal illness states[3].

\section{IMAGE TECHNOLOGY IN MEDICAL APPLICATIONS}

Imaging Technology - Development in picture innovation have shaped the prerequisite to set up whether new advances are viable and cost gainful. This innovation works under the accompanying regions:

Magnetic reverberation imaging of the knee

Computer helped identification in mammography

Endoscopic ultrasound in arranging the esophageal malignancy

Magnetic reverberation imaging in low back torment

Ophthalmic Imaging - This works under two classifications:

1.Improvement of mechanized programming analyzes the retinal pictures to hint at early diabetic retinopathy

2.Improvement of instrumentation - Concentrates on advancement of filtering laser ophthalmoscope.[4]

\section{SOME OF RETINAL DISEASES}

\subsection{Age-Related Macular Degeneration}

Age-related macular degeneration or AMD is an eye clutter and a most essential wellspring of vision misfortune among individuals age 50 and more established and it makes hurt the macular which is little spot close to the focal point of the retina and the part of the eye wanted for sharp, focal vision. AMD influences so gradually that vision misfortune does not happen for quite a while. Here and there sickness advances quicker and may lead to lost vision in one or the two eyes.[4]

Revised Manuscript Received on January 2, 2020.

B.SakthiKarthiDurai, Assistant Professor, CSE department, PSNACET, Dindigul, Tamilnadu, India

J.Benadictraja, Associate Professor, CSE department, PSNACET, Dindigul, Tamilnadu, India

C.Sathya, Assistant Professor, , CSE department, PSNACET, Dindigul, Tamilnadu , India 


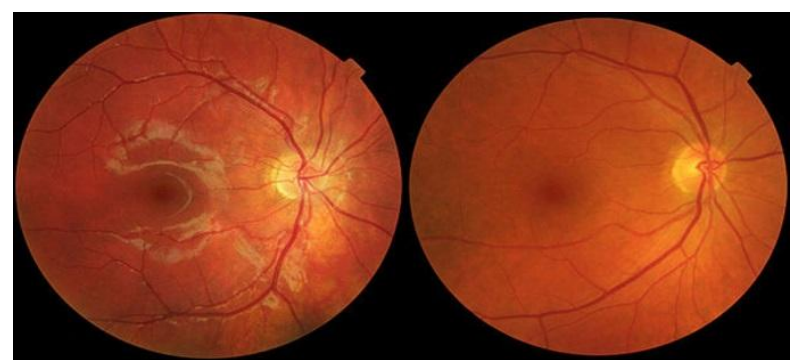

Fig 1. Age related macular degeneration

\subsection{Cataract}

Cataract is one of the main sources of visual impairment in the world. It is the blurring of the focal point that causes loss of vision. Most cataracts are identified with maturing. Other hazard factors diabetes, smoking, presentation to daylight. The retina for the most part contains three sections: optic disc, macular and vein. Optic disc is the most astounding round plate amidst the retina furthermore, ties with mind nerves. Macular is the optical focus of the eye and it is high in lutein which influences it to wind up noticeable yellow. The vein is isolated into conduit and vein. Cataract is grouped into four distinct evaluations: ordinary, mellow, medium and extreme. In the event that the optic disc, enormous vessel and little vessel are clear, the retina picture is an ordinary one. Regardless of whether little vessel isn't clear, it is characterized as a mellow. On the off chance that the little vessel and enormous vessel are unclear, it is said to be medium cataract. In the event that the optic plate isn't clear, it is known as a serious cataract.[4]

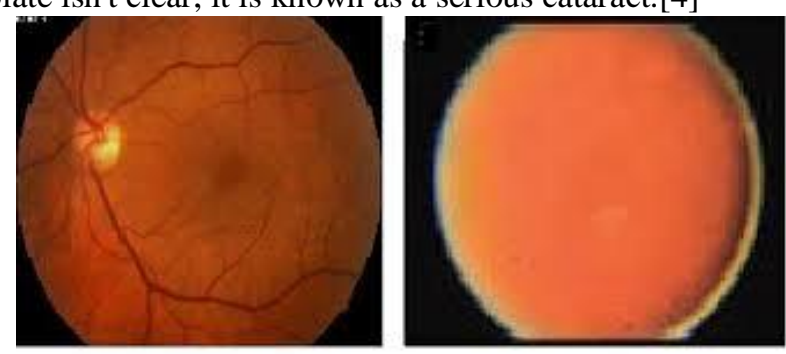

Fig 2:Cataract

\subsection{Glaucoma}

Glaucoma is a ceaseless eye infection in which the optic nerve is logically harmed. The Intra-Ocular Pressure (IOP), which keeps up a lasting state of the human eye and shields it from twist, rises in light of the fact that the right measure of liquid can't deplete out of the eye. A obstructing issue happens inside the seepage channels even the passage to the seepage channels work legitimately. This sort of Glaucoma grows bit by bit and in some cases without clear sight misfortune for a long time and it very well may be treated with meds whenever analyzed at the before stage. Edge Closure Glaucoma happens when the waste channels get blocked. Iris isn't as wide and open as in the standard case. The external edge of the iris groups up over the waste waterways, when the understudy amplifies excessively or too quickly. There are two primary kinds of Glaucoma Primary Open Angle Glaucoma (POAG) and Angle Closure Glaucoma (ACG) . The progressions in the retinal structures which are related with Glaucoma are Optic Nerve Head (ONH) change, Neuroretinal Rim Loss Determination, Retinal Nerve Fiber Layer (RNFL) abandons, Peripapillary Atrophy (PPA).[4]

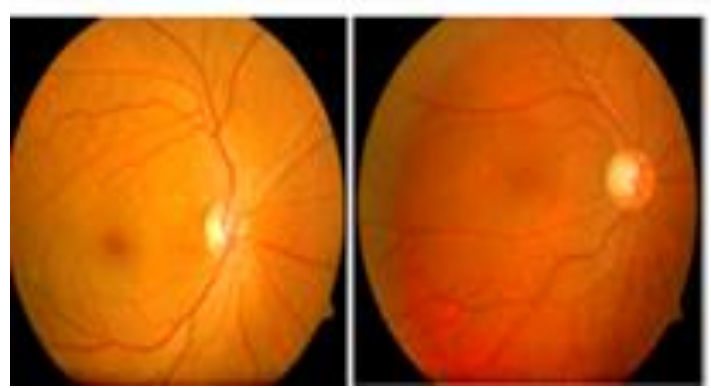

Fig 3:Glaucoma

\section{RETINAL DISEASE DETECTION AND IMAGE PROCESSING}

Retinal Disease discovery finds and portions Retinal Disease locales from jumbled pictures, either acquired from video or still picture. It has various applications in territories like reconnaissance and security control frameworks, content based picture recovery, video conferencing and insightful human PC interface. A large portion of the present Retinal malady acknowledgment frameworks assume that retinal illness is promptly accessible for preparing. In any case, we don't normally get pictures with simply Retinal Disease. We need a framework that will section Retinal Disease in jumbled pictures. With a versatile framework, we can some of the time approach the client to model for the Retinal Disease ID errand. In expansion to making a progressively agreeable target, we can communicate with the framework so as to improve and screen its identification. With a convenient framework, recognition appears to be less demanding. The undertaking of retinal sickness recognition is apparently inconsequential for the human mind, yet regardless it remains a testing and troublesome issue to empower a PC/portable telephone/PDA to do Retinal Disease identification. This is on the grounds that the human Retinal Disease changes as for interior variables like Retinal Disease articulation, facial hair, mustache glasses and so on and it is additionally influenced by outside elements like scale, helping conditions, and differentiation between Retinal Disease, foundation and introduction of Retinal Ailment. [5]

Retinal Disease discovery remains an open issue. Numerous analysts have proposed diverse techniques tending to the issue of Retinal Disease identification. In an ongoing overview Retinal Disease location method is ordered into highlight based and picture based. The element based systems use edge data, skin shading, movement and symmetry measures, highlight investigation, snakes, deformable formats and point dispersion. Picture based systems incorporate neural systems, direct subspace technique like Eigen Retinal Disease, fisher Retinal Disease and so forth. The issue of Retinal Disease discovery in still pictures is all the more difficult and troublesome when contrasted with the issue of Retinal Disease discovery in video since feeling data can prompt likely areas where Retinal Disease could be found.

Image Processing and examination is a strategy and science to identify deteriorated tissue. 
The primary favorable position of medicinal imaging is to make conclusion as conceivable as noninvasive path in the treatment arranging and clinically determination. There are different sorts of restorative imaging advancements dependent on noninvasive methodology like Computed Tomography (CT), Magnetic Resonance Imaging (MRI) and $\mathrm{X}$-Ray etc.MRI is best reasonable brilliant therapeutic imaging innovation instead of others to gather ideal inner data of the body organ for clinical finding. Restorative imaging is a field in which inquires about create instruments to get, control and accomplish advanced pictures that are utilized to give better mind to the patients. In restorative science the issue just as the information stream is three dimensional and the push to take care of the issue is for the most part mix of both human and machine.[5]

Medicinal tasks of image processing can frequently be part into three zones:

1.Data tasks like separating, clamor expulsion, and differentiation and highlight improvement

2.Detection of ailments and occasions.

3.Qualitative investigation of the injury or identified occasions.

\section{IMAGE PROCESSING IN RETINA}

\subsection{Diabetic Retinopathy}

Diabetes mellitus may cause modifications in the retinal microvasculature prompting diabetic retinopathy. Unchecked, propelled diabetic retinopathy may prompt blindness. It can be repetitive and tedious to decode inconspicuous morphological changes in optic disk, micro aneurysms, hemorrhage ,blood vessels, macula, and exudates through manual review of fundus pictures.DR is classified into five types namely mild Non-Proliferative Diabetic Retinopathy(NPDR), moderate NPDR ,severe NPDR ,PDR and Macular Edema (ME).

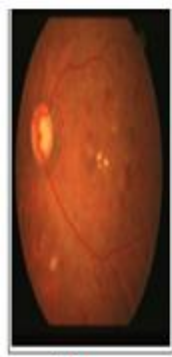

(a)

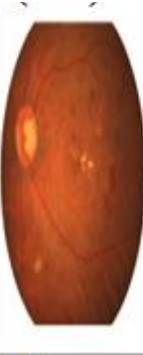

(b)

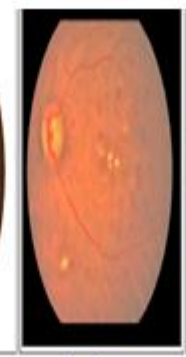

(c)

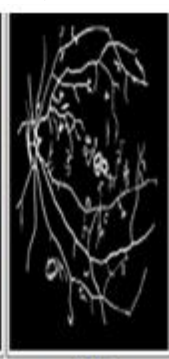

(d)

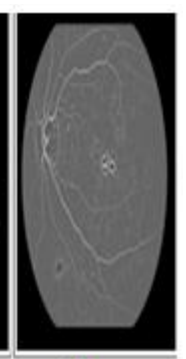

(e)
Fig 4: Images of DR (a)Raw (b)Masked (c)Equalized (d) vessels(e)MSF vessels

The computer Aided Diagnosis(CAD) technique, it describe two systems namely disconnected and connected systems. In the disconnected system, a set of preparing pictures were pre handled to remove features. Further, critical highlights were chosen utilizing separating or wrapper strategies and used to prepare the classifier. In the connected system, only the noteworthy highlights which were chosen amid preparing stage were removed from the testing set. The prepared model groups the test set in to either ordinary or DR class utilizing the noteworthy highlights. Further, the classifier execution was assessed by contrasting the anticipated and highest quality level marks of the test set, which gives the accuracy, sensitivity , and specificity. This segment depicts precise study of programmed analysis of DR utilizing retinal fundus images. The point of the CAD is to recognize typical and DR utilizing highlights like territory of MA, exudates, blood vessels, node points, HEM, textures, etc. [6]

\section{2glaucoma Detection}

Glaucoma is described by harm to the optic nerve through expanding degeneration of the nerve strands. The movement of the malady is asymptomatic in the beginning times yet bit by bit prompts irreversible vision misfortune.

Ophthalmologists utilize three vital strategies to identify beginning of glaucoma. The primary methodology is the evaluation of expanded intraocular weight inside the eye. The sufficiently delicate for early recognition and glaucoma can now and then happen without expanded eye weight. The second methodology distinguishes field of strange vision with particular gear which makes it unsatisfactory for an extensive screening of glaucoma aside from in complex restorative focuses. The third methodology is assessment of harm to the optic nerve. This is most solid yet requires a prepared proficient, is tedious, costly and exceedingly abstract. Master appraisal may change contingent upon experience and preparing CDR values from fragmented optic glass and circle are a vital marker of harm to the optic nerve.

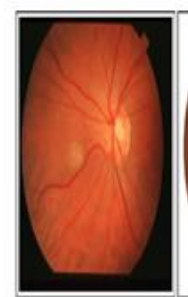

a)

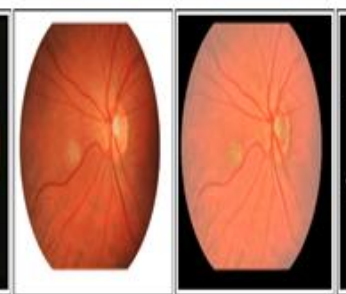

b)

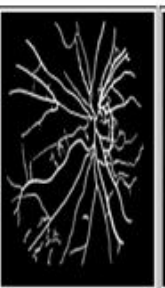

d)

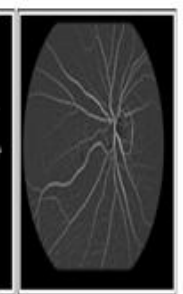

e)
Fig 5:Images of Glaucoma (a)Raw (b)Masked (c)Equalized (d)Vessels (e)MSF vessel

Convolutional can be stretched out to higher dimensional pictures and bits. For the most part pieces will in general be extensively littler than the pictures. Convolution systems abuse three key thoughts scanty communications, parameter sharing and equivariant portrayals. Utilizing convolutional channels as building blocks, complex convolutional systems can be developed. A portion of the imperative building squares of CNNs are: Convolutional filter and max pooling layer.

Convolutional functions between $\mathrm{h}$ and $\mathrm{k}$ can be written as;

$$
\mathrm{x}(\mathrm{k})=\mathrm{h}(\mathrm{t}) * \mathrm{k}(\mathrm{t})=\int_{-\infty}^{w} h(t-\tau) * k(\tau) d \tau
$$

Breaking down each pixel in the trimmed pictures comprises a noteworthy computational weight. Furthermore, data at the pixel level is exceedingly excess since neighboring pixels will in general give exceptionally corresponded data.

This issue is tended to utilizing an entropy based inspecting plan to choose the most educational pixels from the picture. 
Uniform testing with equivalent likelihood leaves behind the chance to extricate significant data for the calculation. In the most pessimistic scenario, the tested focuses spread the picture however neglect to give a complete record of where "intriguing" pat-terns are available. In this way, non uniform examining approaches have been utilized in different applications. Since the proposed strategy depends on convolutional channels, it is critical to choose focuses with instructive environment. Something else, nearby fixes around chosen focuses won't yield adequately discriminative data.[7]

\subsection{Hpersensitive Retinopathy}

Retinal variation from the norm related with high blood weight is known as hypertensive retinopathy (HR). This illness prompts lasting visual impairment so auspicious finding and treatment of the illness is vital. Fundus picture examination is utilized to analyze HR. A robotized framework for the early location of HR turns out to be valuable for ophthalmologists and patients. A variation from the norm of the retina brought about by hypertension is called Hypertensive retinopathy(HR) . Computerized fundus picture examination is utilized to analyze HR by following changes in the pictures. These progressions incorporate convoluted arterioles, hemorrhages, hard exudates, cotton fleece spots and papilledema. A vital manifestation of HR is strangely wide veins prompting low proportion of normal distance across of supply routes to veins (AVR). This infection influences of all grown-ups and individuals more established than 60 years.

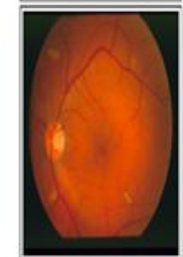

(a)

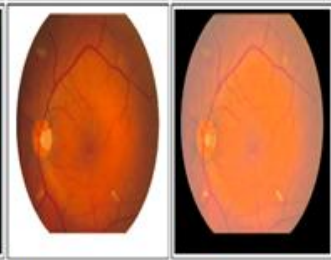

(b)

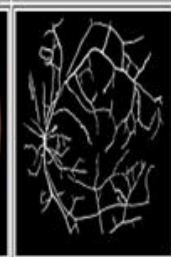

(d)

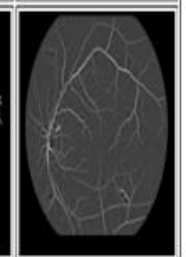

(e)
Fig 6:Images of HR (a) Raw (b)Masked (c)Equalized (d)vessels (e)MSF

The location of HR utilizing arteriovenous (AV) proportion. The framework comprises of novel strategy for characterization of vessels as arteries and veins utilizing new feature vector and hybrid classifier. This methodology deals with vessel extraction display,vessel order model, AVR computational model, ROI Detection demonstrate. In vessel extraction display Gabor wavelet used for enhancement and segmentation is done with the help of multilayered thresholding.In vessel order model vector formulation and hybrid classifier are used.AVR is used for grading of HR.ROI detection model is used for extraction of optic disc.[8]

\subsection{Age -Related Macular Degeneration}

AMD is the fourth driving reason for visual deficiency around the world. On the off chance that it isn't identified in beginning periods it causes misfortune of vision. AMD is a typical eye condition and a main reason for vision misfortune among individuals age 50 and more established. It causes harm to the macula, a little spot close to the focal point of the retina furthermore, the piece of the eye required for sharp, focal vision. AMD influences the macula, the piece of the eye that enables you to see fine detail. The macula is comprised of a large number of light-detecting cells that give sharp, focal vision. At the point when the macula is harmed, the focal point of your field of view may seem hazy, twisted, or dim. There are two kinds of AMD, wet and Dry what's more, wet macular degeneration influences the retina because of filling of liquid under the retina .it prompts draining and scarring causing loss of vision. In Dry macular degeneration beginning indications are the nearness of greasy stores called drusen on the retina. Most of the general population with macular degeneration are influenced by the dry kind. AMD without anyone else's input does not lead to finish visual deficiency. Be that as it may, the loss of focal vision in AMD can meddle with exercises, for example, the capacity to see faces, drive, read, compose.

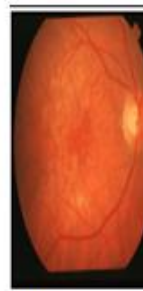

(a)

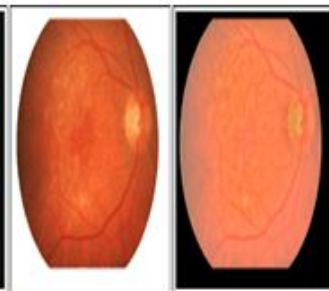

(b)

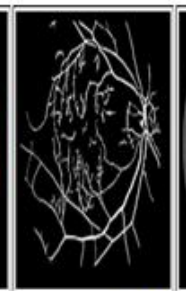

(d)

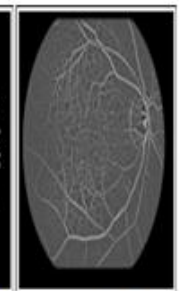

(e)
Fig 7: Images of ACM (a) original (b) Masked (c)Equalized (d)vessel(e)MSF vessel

The k-means algorithm is used for classification and the adaptive histogram equalization is followed for preprocessing. feature extraction includes optic disk selection and drusen detection. Adaptive histograms equalization is a PC picture preparing procedure used to improve differentiates in pictures. It is appropriate for improving the nearby difference and upgrading the meanings of edges in every district of a picture. Wavelet change is the way toward removing critical data from a picture. Highlights of the retinal pictures are removed from the changed coefficients. The wavelet change is an amazing and adaptable device that has been connected to a wide range of picture preparing issues, for example, picture coding, surface investigation and shape examination. Nonstop Wavelet Transform (CWT) in at least two measurements is a very productive and adaptable instrument in picture investigation. After histogram thresholding, smoothing and edge location the picture is divided.]For characterizing every pixel into vessel or not, the KNN is connected with the estimations of these pixels. [9]

\section{COMPARSION OF RETINAL DISEASE IDENTIFICATION METHODS}

\begin{tabular}{|c|c|c|}
\hline $\begin{array}{l}\text { RETINAL } \\
\text { DISEASES }\end{array}$ & $\begin{array}{l}\text { ORIGINAL } \\
\text { IMAGES }\end{array}$ & ALGORITHM \\
\hline $\begin{array}{l}\text { Diabetic } \\
\text { retinopathy }\end{array}$ & & $\begin{array}{l}\text { (1)Machine learning } \\
\text { includes Gaussian } \\
\text { Mixture model (GMM), } \\
\text { k-nearest neighbor } \\
\text { (kNN), support }\end{array}$ \\
\hline $\begin{array}{l}\text { Published By: } \\
\text { Blue Eyes Intell } \\
\text { \& Sciences Pub }\end{array}$ & Engineering & novation \\
\hline
\end{tabular}




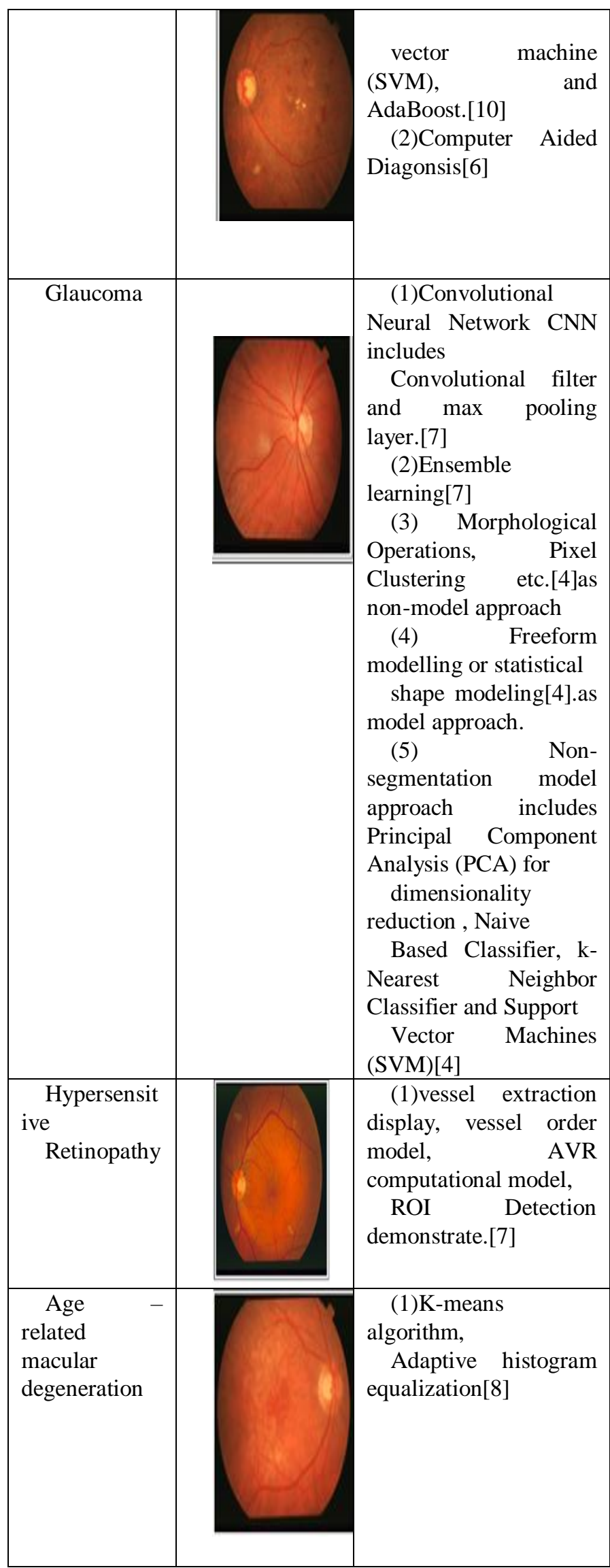

\section{CONCLUSION}

Thus the image processing plays an important role in medical field especially in eye which comes major part of human being. The methods and type of images gives detail description to both medical and scientific field to address of issues in world with solutions. This survey defines the real dataset of affected patients and the learning algorithm used.

\section{ACKNOWLEDGMENT}

The authors would like to thank the Management of PSNA College of Engineering and Technology, Dindigul for the support to complete this project inside the campus by providing Lab facility.

\section{REFERENCES}

1. AsmaYasrib\&Mohd Adam Suhaimi” Image Processing in Medical Applications,"published in Journal of Information Technology Impact, 2003

2. Renato Besenczi, Janos Toth, AndrasHajdu,"A review on automatic analysis techniques for color fundus photographs "published in Computational and Structural Biotechnology Journal 14 (2015) 371384

3. https://www.embs.org/about-biomedical-engineering/our-areas-ofresearch/biomedical-imaging-image-processing/

4. Anjali K, Bhavya K Bharathan, Beena M V," Survey on Retinal Disease detection techniques" published in International Journal of Advanced Research in Computer Engineering \& Technology (IJARCET), Volume 6, Issue 1, January 2017

5. Parul1, NeetuSharma,"A Study on Retinal Disease Classification and FilterationApproaches", published in International Journal of Computer Science and Mobile Computing, A Monthly Journal of Computer Science and Information Technology ,IJCSMC, Vol. 4, Issue. 5, May 2015,pg.158 - 165

6. MuthuRamaKrishnanMookiaha,n, U.RajendraAcharyaa,b, ChuaKuangChua a, ChooMinLim a, E.Y.K.Ng c, AugustinusLaude,"Computeraideddiagnosisofdiabeticretinopathy:Are view"published in Computers inBiologyandMedicine43(2013)21362155

7. Julian Zilly a, Joachim M. Buhmannb, DwarikanathMahapatrab,,"'Glaucoma detection using entropy sampling and ensemble learning for automatic optic cup and disc segmentation ",published in Computerized Medical Imaging and Graphics 55 (2017) 28-41

8. SarmadKhitran, M. UsmanAkram, AnamUsman, UbaidullahYasin, "Automated System for the Detection of Hypertensive Retinopathy",published in IEEE conference,2015

9. PoonamBhosale, PradnyaKulkarni,SarikaBobde,"Automatic Detection Of Age Related Macular Degeneration Using Retinal Colour Images", published in Sixth Post Graduate Conference for Computer Engineering (cPGCON 2017) Procedia ,International Journal on Emerging Trends in Technology (IJETT)

10. DREAM: Diabetic Retinopathy Analysis Using Machine LearningSohiniRoychowdhury, Student Member, IEEE, Dara D. Koozekanani, Member, IEEE, andKeshab K. Parhi, Fellow, ieee, ieee journal of biomedical and health informatics, vol. 18, no. 5, september 2014

\section{AUTHORS PROFILE}

B.SakthiKarthiDurai, Assistant Professor, CSE department, PSNACET, Dindigul, Tamilnadu, India.

J.Benadictraja, Associate Professor , CSE department, PSNACET, Dindigul, Tamilnadu , India.

C.Sathya, Assistant Professor, , CSE department, PSNACET, Dindigul, Tamilnadu , India 